\title{
Electric Vehicle Charging Reservation Under Preemptive Service
}

\author{
Yue Cao, Shuohan Liu, Ziming He, Xuewu Dai, Xiaoyan Xie, Ran Wang, Shengping Yu
}

\begin{abstract}
Electric Vehicles (EV) are environment-friendly with lower $\mathrm{CO} 2$ emissions, and financial affordability (in term of battery based refuel) benefits. Here, when and where to recharge are sensitive factors significantly impacting the environmental and financial gains, these are still challenges to be tackled. In this paper, we propose a sustainable and smart EV charging scheme enables the preemptive charging functions for heterogeneous EVs equipped with various charging capabilities and brands. Our scheme intents to address the problems when EVs are with various ownerships and priority, in related to the services agreed with charging infrastructure operators. Particularly, the anticipated EVs' charging reservations information with heterogeneity (are multiscale) including their EV type, expected arrival time and charging waiting time at the charging stations (CSs), have been considered for design, planning and optimal decision making on the selection (i.e., where to charge) among the candidature CSs. We have conducted extensive simulation studies, by taking the realistic Helsinki city geographical and traffic scenarios as an example. The numerical results have confirmed that our proposed preemptive approach is better than the First-Come-First-Serve (FCFS) based system, associated with its significant improvement on the reservation feature in $\mathrm{EV}$ charging.
\end{abstract}

\section{INTRODUCTION}

Electric Vehicles (EVs) [1] have been key component to achieve the green transport. The work already been completed tends to analyse when to charge EVs (charging scheduling) to save cost of charging and minimize peak loads and flatten aggregated demands [2]-[6]. Different from them addressing "when/whether", charging process should occur while they have been parked at homes/Charging Stations (CSs) (namely charging scheduling), similarly interest addresses "where" EVs should be charged while they are on-the-move during journeys (namely CS-selection). Here, an on-the-move EV requests charging service, needs to move towards an appropriate CS for charging process. Due to the long charging time (experienced by existing charging technologies), to optimally plan where to charge has become a critical issue.

We refer to the charging system widely adopted by literature [5], [7], which relies on a Global Aggregator (GA) to manage each real-time EV charging request/reply in a centralized manner. Normally, the cellular network, e.g., 3G/Long Term

Y.Cao and S.Liu are with the School of Computing and Communications, Lancaster University, UK. Email: yue.cao@lancaster.ac.uk; S.Liu is with the Newcastle University, UK; Z.He is with the VIAVI Solutions, UK; X.Dai and S.Yu are with the State Key Laboratory of Synthetical Automation for Process Industries, Northeastern University, China; X.Xie is with the LVMT, UMR-T 9403, Ecole des Ponts ParisTech, IFSTTAR, UPEM, UPE, 77455, Champssur-Marne, France; R.Wang is with the College of Computer Science and Technology, Nanjing University of Aeronautics and Astronautics, China.

This work is partially supported by National Natural Science Foundation of China (61773111, U1864206, and 61790574).
Evolution (LTE) is applied for ubiquitous and seamless communication. The GA monitors CSs' condition (the number of EVs being parked and their charging time), and implements the charging management optimization (including both charging scheduling and CS-selection).

In literature, the CS-selection schemes based on the closest distance [8] and minimum queuing time [7], [9]-[11] have been studied. Nevertheless, none of them has adequately investigated the influence of preemptive EV charging scheduling on actual decision making. Regarding heterogeneities of EVs, those EVs with different types (depending on ownership, e.g., citizens and police, as well as charging capabilities, e.g., maximum battery volume and electricity consumption) are eligible for preemptive charging. For example, an EV owned by military or police with emergence will preempt the charging service, prior to others (owned by citizens) already been parked [12], [13]. In this situation, an incoming EV might be scheduled for charging, prior to those already being parked at a CS. This normally happens when the incoming EV is with a higher charging priority.

Inevitably, a potential charging hotspot may happen if more EVs travel towards a similar CS for charging, due to the fact that the CS-selection decision just considers CSs' local condition. In this context, it is suggested that EVs should further report their charging reservations [1], [14]-[16]. These anticipated information together with the CS's local condition (e.g., available time for charging), will be used to estimate the congestion status of CS in a near future.

However, there is still vacancy to integrate charging reservation for preemptive charging service, to balance the charging demands among CSs and particularly reduce the time that heterogeneous EVs wait for charging. Inevitably, the preemptive charging scheduling strategy will introduce new design on CS-selection. This is because the GA must know whether a reserved EV's charging service will be preemptive, and makes optimal recommendation on CS-selection.

Compared to the works enabling charging reservation service, a fundamental difference between the proposed work and [15] is that, the latter analyse the scenarios of a highway where the EV has to pass through all CSs on its way. The expected waiting time for EV is calculated, by jointly considering the charging waiting time at a CS that the EV needs charging for the first time, and that of any consequent CS before exiting the highway. In the proposed scenario, targeted city scenario the EV will select a single geographically distributed CS for charging and, waiting time is considered only in respect to a certain CS. Previous works on CS-selection (even with charging reservation enabled [1], [14]-[16]) rarely consider the 
preemptive EV charging scheduling (when/whether to charge), while they are just based on the First Come First Serve (FCFS) charging scheduling strategy.

In the light of this, we summarize our contributions over literature works, by answering the following questions:

What is the impact of preemptive charging based CSselection? The proposed CS-selection scheme considers the underlying preemptive charging scheduling, where the charging of incoming EVs (with a higher charging priority) may take place prior to others (with a lower charging priority) already been parked at a CS.

What is the benefit to bring heterogenous EVs' charging reservations? Upon that, the proposed CS-selection scheme requires EVs (with prioritized charging nature) to further report their charging reservations to the GA (through cellular communication). Such anticipated information from EVs (which are heading to their selected CSs), will be reported to the GA for CSs' condition estimation in the near future.

\section{PRELIMinary}

\section{A. System Cycle for Preemptive EV Charging}

The Fig. 1 describes system cycle:

Driving Phase: The EV is on journey, with sufficient battery energy above the level of set threshold for State of Charge (SOC).

Charging Planning Phase: The EV sends a charging request to GA and move towards a $\mathrm{CS}$ for charging operation.

- With basic charging service, the GA replies the charging planning back to the EV, then the EV travels towards that selected CS for charging.

- With reservation enabled charging service, upon accepting the charging planning from GA, the EV sends its charging reservation request to the GA. This procedure refers to Charging Reservation Phase.

Charging Scheduling Phase: The parked EV will wait CS to schedule its charging slot, upon the arrival of EV at the CS.

Battery Charging Phase: Once EV is being charged, it will turn to the Driving Phase when its battery is charged fully.

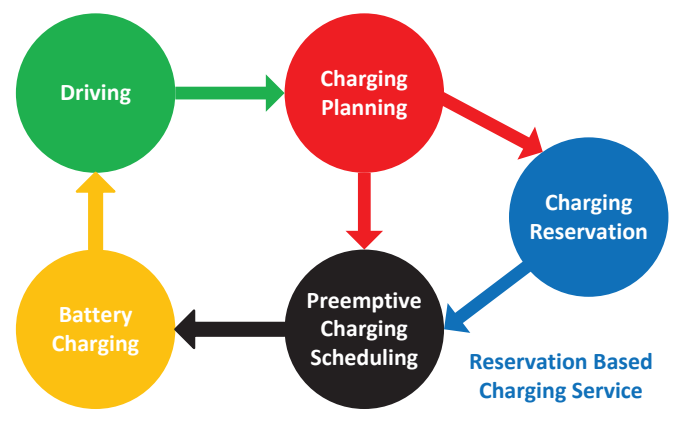

Fig. 1. System Cycle of Preemptive EV Charging

\section{B. Preemptive Charging Scheduling For Parked EVs}

Our main focus is on the CS-selection (concerning where to charge). To specify the operations of the system clearly, we first present the underlying charging scheduling scheme (concerning when to charge).

Each CS ranks the charging priority of parked EVs, and applies multiple charging slots to process charging in parallel. Two type of EVs are considered, that are "High PrioritizedEV (H-EV)" and "Low Prioritized-EV (L-EV)" respectively. In general, we differentiate EVs depending on their unique charging capabilities (e.g., ownership, brand, maximum battery volume and electricity consumption). A policy for their charging scheduling is given as follows:

- Those EVs with the "H-EV" type are normally scheduled prior to those with the "L-EV" type, regardless of their arrival time at a CS. This policy guarantees a preemptive charging service for those "H-EVs". Note that H-EVs (either been parked or just arrive) can preempt L-EVs (parked but have not being charged).

- The First Come First Serve (FCFS) order for those with the same type and, prioritized in such way that the one arrived earlier is given priority. Note that the FCFS is commonly applied by most previous works for charging planning [14], [15].

\section{Preemptive EV Charging Reservation}

\section{A. Estimation of Earliest Available Time For Charging (EATC)}

Mainly two type of queues are defined here; The EVs currently in the process of charging contained the queue of $N_{C}$, while those in the waiting position are placed in the queue of $N_{W}$. In the case presented in Algorithm 1, the current time in network as denoted by $T_{\text {cur }}$, is also known as the earliest available charging point, if all charging slots are unoccupied at a specific time. Such case means the CS is currently available for charging.

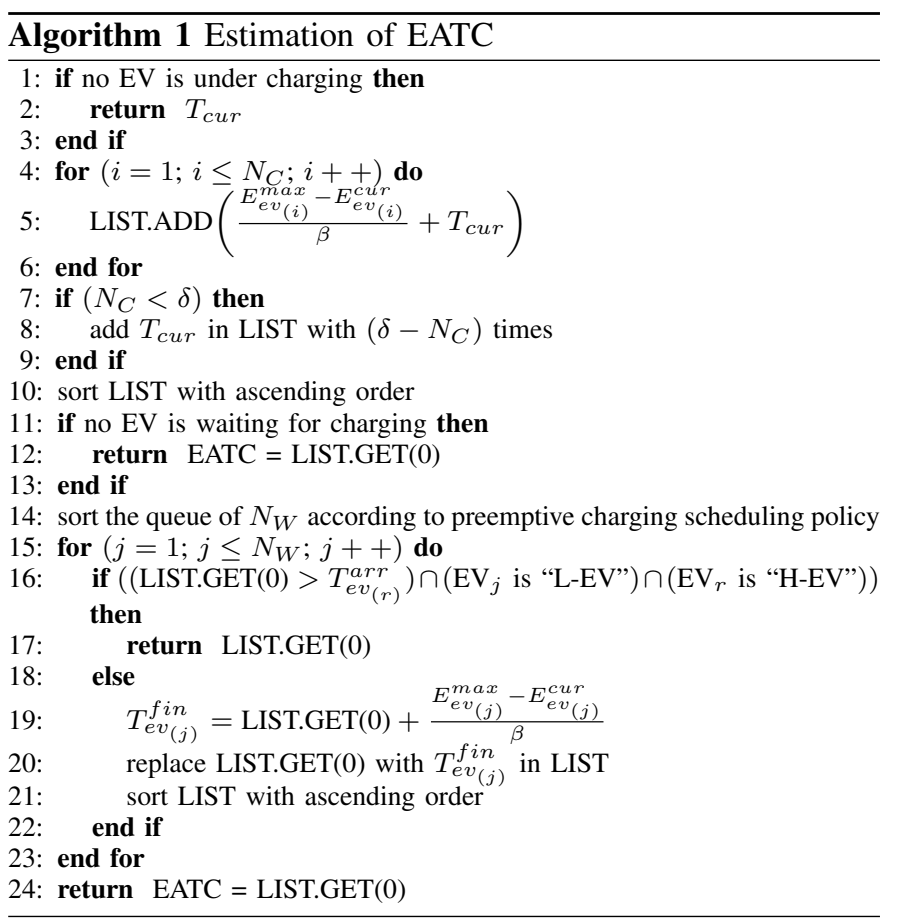


Starting from line 4 , the time duration $\frac{E_{e v}^{\max }-E_{e v}^{c u r}(i)}{\beta}$ to fully recharge the battery of each $\mathrm{EV}_{i}$ (in the queue of $N_{C}$ ), will be summated with $T_{\text {cur }}$. The summation of these two value reflects when the charging for $\mathrm{EV}_{i}$ will be finished. Furthermore, this summation will be added into LIST, which reflects that a charging slot will be available at $\left(\frac{E_{e v}^{\max }-E_{e v}^{c u r}}{\beta}+T_{c u r}\right)$, recall that $\left(\frac{E_{e v_{(i)}}^{\max }-E_{e v(i)}^{c u r}}{\beta}\right)$ is the time to fully recharge $\mathrm{EV}_{i}$.

Upon the above process, the following presentation between lines 7 and 9 implies that not all charging slots are fully occupied, because there are still $\left(\delta-N_{C}\right)$ charging slots available for charging. In this context, $T_{c u r}$ is the estimated available charging time for unoccupied charging points.

Here, Algorithm 1 will return the EATC, either if there is no EV waiting for charging (the condition at line 11), or a loop operation for each $\mathrm{EV}_{j}$ (in the queue of $N_{W}$ ) waiting for charging has been processed (between lines 15 and 23).

In the other case, the loop starts from sorting the queue of $N_{W}$, which is based on preemptive charging scheduling technique. Meanwhile, the LIST concerned with EVs in the charging phase is put in ascending order, which means that the earliest available charging slot is placed at the top of LIST. Therefore, we denote LIST.GET(0) as the first value in LIST:

- In particular, since $\mathrm{EV}_{r}$ (the on-the-move $\mathrm{EV}$ needs charging service) with the "H-EV" type, could preempt charging prior to those parked $\mathrm{EV}_{j}$ (in the queue of $N_{W}$ ) with the "L-EV" type, the EATC is returned at line 17, given:

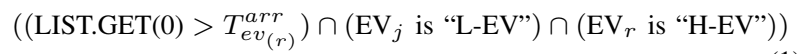

Above condition as highlighted at line 16, implies that the arrival time of $\mathrm{EV}_{r}$ is earlier than the LIST.GET(0), meanwhile the processed $\mathrm{EV}_{j}$ (in the queue of $N_{W}$ ) is with the "L-EV" type. As such, the charging of parked $\mathrm{EV}_{j}$ will be preemptive by the incoming $\mathrm{EV}_{r}$.

- Apart from the above special case, the operation at line 19 calculates the charging finish time $T_{e v_{(j)}}^{f i n}$ of each $\mathrm{EV}_{j}$, and replaces this value with LIST.GET(0). Upon the above, the LIST will be further sorted with ascending order, such that LIST.GET(0) is updated for further calculation.

The above loop operation ends when all $\mathrm{EV}_{j}$ have been processed, then the EATC (the first value in LIST) is returned at line 24.

\section{B. Charging Reservation with EV Heterogeneity}

Every EV sends its charging reservation status to GA. The reservation information is useful for the GA to analyse CS condition in the near future, like Expected Waiting Time (EWT), such that a potential charging hotspot could be alleviated. Here, the CS with the minimum EWT is selected for $\mathrm{EV}_{r}$ (the EV needs charging service). Major computation logic is illustrated through Algorithm 2 and Algorithm 3.

The charging reservation generated from the $\mathrm{EV}$ (e.g., $\mathrm{EV}_{r}$ ) which is travelling towards the selected CS, is reported via the cellular network in this section. It normally includes: $\langle$ EV ID $\rangle$ : The ID of EV which needs charging and has been replied with the selected $\mathrm{CS}$.

$\langle$ EV Type $\rangle$ : Either with "L-EV" or "H-EV" type.

$\langle$ Selected CS $\rangle$ : Where the EV will travel for charging.

$\langle$ Arrival Time $\rangle$ : Based on the travelling time $T_{e v}^{t r a}$ calculated from the current location of EV to that CS via the shortest road path, the expected arrival time $T_{e v}^{a r r}$ is given by:

$$
T_{e v}^{a r r}=T_{c u r}+T_{e v}^{t r a}
$$

Here, the detour issue is not considered.

$\langle$ Expected Charging Time $\rangle$ : we denote $T_{e v}^{c h a}$ as the expected charging time upon that arrival, where:

$$
T_{e v}^{c h a}=\frac{E_{e v}^{\max }-E_{e v}^{c u r}+S_{e v} \times T_{e v}^{t r a} \times \alpha}{\beta}
$$

Note that $S_{e v} \times T_{e v}^{t r a} \times \alpha$ is the amount of electric energy consumed for travelling, where $S_{e v}$ is the EV speed and $\alpha$ is the per meter energy consumption.

\section{Estimation of Expected Waiting Time (EWT)}

The estimation of EWT at a CS depends on two cases:

- Case-1: In the first case detailed by Algorithm 2, we consider that incoming $\mathrm{EV}_{r}$ (only with the "H-EV" type), has chance to get preemptive charging upon its arrival, prior to those "L-EVs" already been parked at a CS.

- Case-2: In the second case detailed by Algorithm 3, we consider that $\mathrm{EV}_{r}$ (regardless of its type) will be charged, either if all EVs (parked at a CS) have been charged, or there is no other EV being scheduled.

1) Case-1: Initially, $\mathrm{EV}_{r}$ is added into the queue of $N_{R}$, meanwhile these parked EVs (in the queue of $N_{W}$ ) are sorted with preemptive charging priority. Algorithm 2 then starts from finding those EVs (in the queue of $N_{W}$ ), by referring to the operations in Algorithm 1. In particular, if the number of EVs (in the queue of $N_{C}$ or $N_{W}$ ) is 0 , the EWT is returned by Algorithm 3 (case-2) at lines 6 and 16 respectively.

Initially, the LIST containing the time slot (about when the charging of those EVs in the queue of $N_{C}$ ) will be finished, is sorted with ascending order at line 14 . The motivation behind this is to obtain the earliest available time for charging, as denoted by LIST.GET(0). Before processing each $\mathrm{EV}_{j}$ (in the queue of $N_{W}$ ) waiting for charging, those $\mathrm{EV}_{k}$ (in the queue of $N_{R}$ ) which have made reservations are initially checked at line 19. This considers the case that, the $\mathrm{EV}_{k}$ with the " $\mathrm{H}$ EV" type and an earlier arrival time $T_{e v_{(k)}}^{a r r}$ than LIST.GET(0), would be charged prior to $\mathrm{EV}_{j}$.

The preemptive charging happens only when $\mathrm{EV}_{j}$ is with the "L-EV" type. In this context, given the condition:

$$
\left(\left(\mathrm { EV } _ { k } \text { is "H-EV") } \cap \left(\mathrm{EV}_{j} \text { is "L-EV") } \cap\left(\operatorname{LIST} \cdot G E T(0)>T_{e v}^{a r r}(k)\right)\right.\right.\right.
$$

at line 20 in Algorithm 2, we have:

- Algorithm 2 will directly return the EWT given by (LIST.GET(0) $-T_{e v_{(r)}}^{a r r}$ ) at line 22, only if the $\mathrm{EV}_{k}$ (being processed in current loop) is the $\mathrm{EV}_{r}$ (the on-the-move EV needs charging).

- Otherwise, from lines 24 to 27, the charging finish time $T_{e v_{(k)}}^{f i n}$ of those $\mathrm{EV}_{k}$ (other than $\mathrm{EV}_{r}$ ) will be replaced 


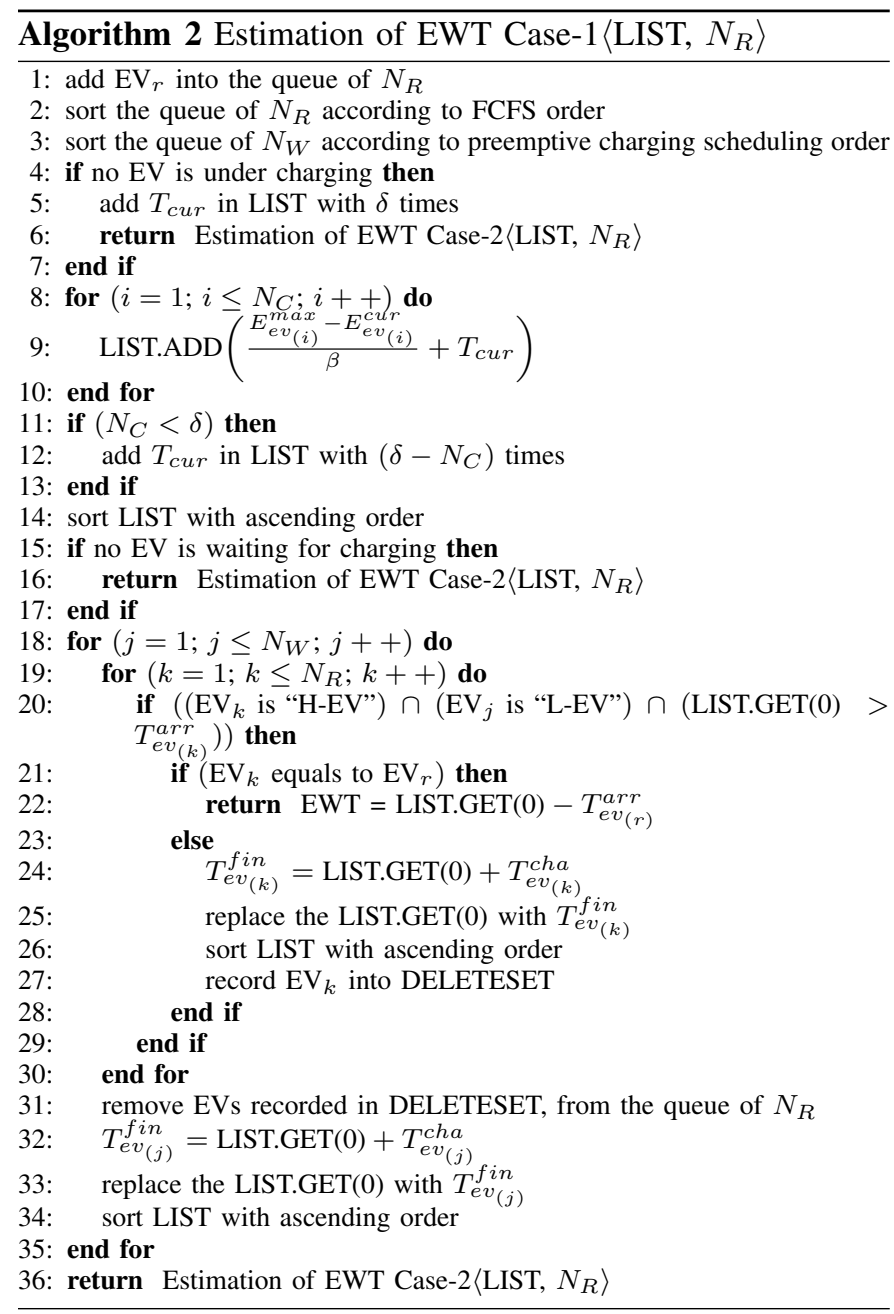

with LIST.GET(0). This implies the preemptive charging of $\mathrm{EV}_{k}$ takes place earlier than $\mathrm{EV}_{j}$. Upon processing each $\mathrm{EV}_{k}$ in current loop, the LIST will be further sorted with ascending order, in order to obtain the updated LIST.GET(0) in next loop. Besides, at line 31, this given $\mathrm{EV}_{k}$ (which involves the updating of LIST) will be removed from the queue of $N_{R}$, since its charging has already been scheduled.

Unless the above loop operations related to $\mathrm{EV}_{k}$ have been processed, those $\mathrm{EV}_{j}$ waiting for charging will be processed. This implies that the charging of $\mathrm{EV}_{j}$ will be started, once any $\mathrm{EV}_{k}$ (meets the condition at line 20) has been charged. Here, the charging finish time $T_{e v_{(j)}}^{f i n}$ also involves LIST update (between lines 32 and 34), until the charging of the last $\mathrm{EV}_{j}$ (in the queue of $N_{W}$ ) has been scheduled. Finally, at line 36, Algorithm 3 is applied, if the charging of $\mathrm{EV}_{r}$ has not been scheduled in previous steps.

2) Case-2: Previously, the inputs of Algorithm 3 including LIST and the queue of $N_{R}$, have already been updated by Algorithm 2. It is highlighted that the input $N_{R}$ excludes those incoming "H-EVs" (which got preemptive charging prior to those parked "L-EVs" at a CS, processed at line 31 in Algorithm 2).

At line 1 of Algorithm 3, we insert the rest of those EVs (in the queue of $N_{R}$ from Algorithm 2), into a newly defined

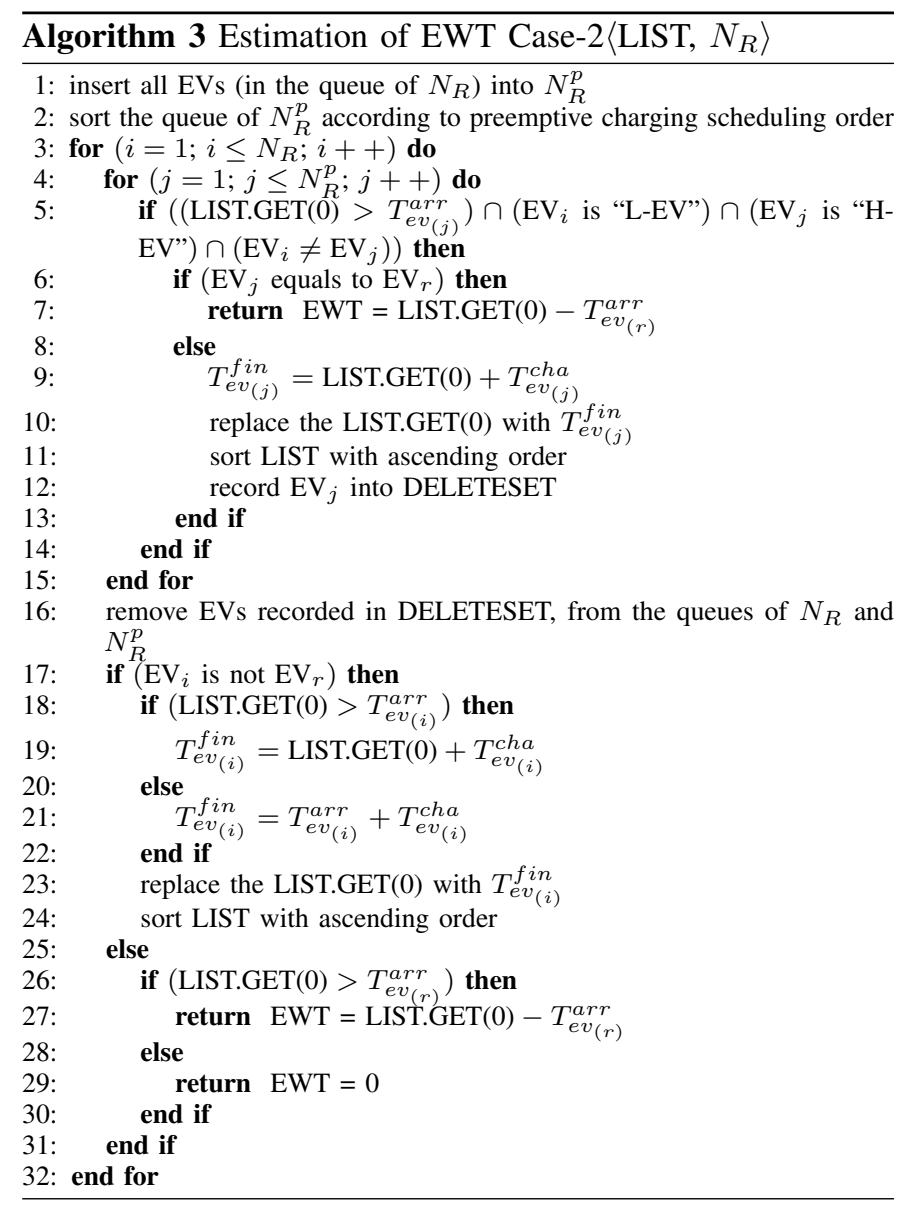

queue $N_{R}^{p}$. Those EVs (in the queue of $N_{R}^{p}$ ) will be sorted, following the preemptive charging scheduling described in Section II-B. This is different from those in the queue of $N_{R}$ following the FCFS order.

For each loop, those $\mathrm{EV}_{j}$ (in the queue of $N_{R}^{p}$ ) with the "H-EV" type and an earlier arrival time than LIST.GET(0), will be charged prior to $\mathrm{EV}_{i}$ (in the queue of $N_{R}$ ) with the "L-EV" type. As such, at line 5, given the condition:

$$
\begin{aligned}
& \left(( \operatorname { L I S T } . G E T ( 0 ) > T _ { e v } ^ { a r r } ) \cap \left(\mathrm{EV}_{i}\right.\right. \text { is "L-EV") } \\
& \left.\cap\left(\mathrm{EV}_{j} \text { is " } \mathrm{H}-\mathrm{EV}^{\prime}\right) \cap\left(\mathrm{EV}_{i} \neq \mathrm{EV}_{j}\right)\right) \text {, }
\end{aligned}
$$

we have:

- At line 7, the EWT will be returned as (LIST.GET(0) $\left.T_{e v_{(r)}}^{a r r}\right)$, if $\mathrm{EV}_{j}$ in the current loop is $\mathrm{EV}_{r}$. Note that Algorithm 3 considers that $\mathrm{EV}_{r}$ was not scheduled for charging, through Algorithm 2.

- At line 9, alternatively the charging finish time $T_{e v_{(j)}}^{f i n}$ of those $\mathrm{EV}_{j}$ (other than $\mathrm{EV}_{r}$ ) will be replaced with LIST.GET(0). This means that the charging of $\mathrm{EV}_{j}$ will take place earlier than $\mathrm{EV}_{i}$.

From lines 9 to 12 , upon processing each $\mathrm{EV}_{j}$, the LIST will be sorted and updated with ascending order. Besides, the given $\mathrm{EV}_{j}$ (which involves the updating of LIST), will be removed from the queues of $N_{R}$ and $N_{R}^{p}$ respectively, as presented at line 16. This is because the $\mathrm{EV}_{j}$ has been already taken into account for the estimation of EWT. Note that both $\mathrm{EV}_{j}$ (in the queue of $N_{R}^{p}$ ) and $\mathrm{EV}_{i}$ (in the queue of $N_{R}$ ) are those EVs 
made reservations, however they are placed into queues sorted with different orders. As such, any $\mathrm{EV}_{i}$ mapping to $\mathrm{EV}_{j}$ that is excluded from line 16, will be skipped for the loop operations at line 3 and 4 respectively.

Further to above, from line 17, $\mathrm{EV}_{i}$ in the current loop will then be processed, once the loop operations between lines 5 and 15 have been finished. This means that the charging of $\mathrm{EV}_{i}$ can only be scheduled, once any $\mathrm{EV}_{j}$ (meets the condition at line 5) has been charged. Here, the arrival time of $\mathrm{EV}_{i}$ (other than $\mathrm{EV}_{r}$ ) will be compared with LIST.GET(0):

- In one case, given (LIST.GET(0) $>T_{e v_{(i)}}^{a r r}$ ), the charging finish time $T_{e v_{(i)}}^{f i n}$ of $\mathrm{EV}_{i}$ is calculated by $\left(T_{e v_{(i)}}^{f i n}=\right.$ LIST.GET $\left.(0)+T_{e v_{(i)}}^{c h a}\right)$ at line 19. This means that the charging slot has not been available upon the arrival of $\mathrm{EV}_{i}$, thus the time to start charging $\mathrm{EV}_{i}$ is LIST.GET(0).

- In another case, we have $\left(T_{e v_{(i)}}^{f i n}=T_{e v_{(i)}}^{a r r}+T_{e v_{(i)}}^{c h a}\right)$ at line 21, where the time to start charging $\mathrm{EV}_{i}$ is $T_{e v}^{a r r}$. This implies that there is an available slot free for charging upon the arrival of $\mathrm{EV}_{i}$, because of (LIST.GET(0) $\left.\leq T_{e v_{(i)}}^{a r r}\right)$.

Then the $T_{e v_{(i)}}^{f i n}$ will be further replaced with LIST.GET(0), similar to the LIST updating as previously mentioned.

Finally, Algorithm 3 will return the EWT once the $\mathrm{EV}_{i}$ in current loop equals to $\mathrm{EV}_{r}$. Then the arrival time of $\mathrm{EV}_{r}$ will be compared with the earliest available time for charging as given by LIST.GET(0). Here, either (LIST.GET(0) $\left.-T_{e v_{(r)}}^{a r r}\right)$ or 0 , is calculated as the expected waiting time for $\mathrm{EV}_{r}$ at lines 27 and 29. This mainly depends on whether a charging slot will be available, upon the arrival of $\mathrm{EV}_{r}$.

\section{Performance Evaluation}

The performance is evaluated via the Opportunistic Network Environment (ONE), under the Helsinki city with an area of $4500 \times 3400 \mathrm{~m}^{2}$ in Fig. 2. Each EV is assigned with a random destination in scenario throughout simulation, and this repeat once EV has reached the SOC threshold. The driving route towards destination is based on the shortest path. $240 \mathrm{EVs}$ with $[2.7 \sim 13.9] \mathrm{m} / \mathrm{s}$ variable moving speed are initialized in the network, with configuration of maximum battery volume, maximum travelling distance, and the SOC threshold under which EV should seek charging: Coda Automotive $\{33.8$ kWh, 193 km, 30\%\} for 120 L-EVs; Hyundai BlueOn $\{16.4$ $\mathrm{kWh}, 140 \mathrm{~km}, 50 \%\}$ for $120 \mathrm{H}-\mathrm{EV}$ s. As such, EVs are differentiated by their brands throughout our simulation. There are totally 7 CSs deployed in the city, with large enough electric energy, each CS is equipped with 5 charging slots and $62 \mathrm{~kW}$ fast charging power.

Unless mentioned, incoming EVs are scheduled based on the preemptive charging policy, as detailed in Section II-B. Systems are compared: Preemptive Charging Without Reservation (PCWR): The GA selects the CS with the minimum value of EATC (calculated in Algorithm 1); Reservation: The reservation system based on FCFS charging scheduling [14]. Proposed: The proposed solution with EWT, as two cases detailed in Algorithm 2 and Algorithm 3.

Throughout the simulation, we fix the number of L-EVs, but only vary the number of H-EVs. Evaluation metrics: Average Waiting Time: As the metric at the EV side, the average

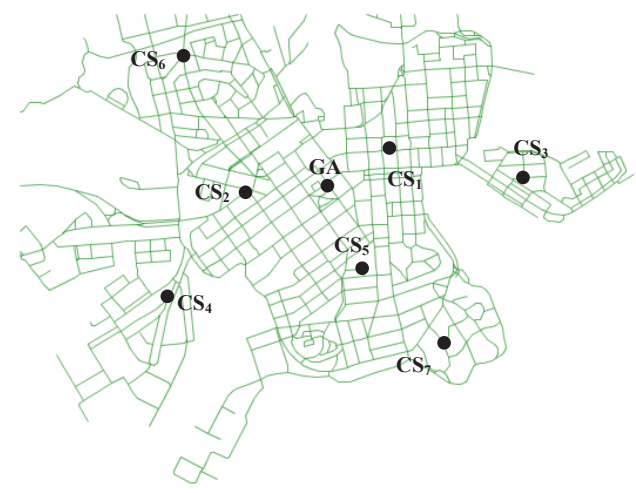

Fig. 2. Simulation Scenario of Helsinki City

waiting time measures the average period between the time that H-EVs/L-EVs arrive at the selected CSs and the time they finish recharging batteries; Number of Charged EVs: As the metric at the CS side, the number reflects the total number of fully charged L-EVs/H-EVs.

In Fig. 3(a), the proposed scheme achieves a shorter average waiting time for H-EVs, than the PCWR. This is because the former estimates the EWT considering EVs' charging reservations, thus the CS condition can be predicted in a near future. With this knowledge, the proposed scheme is able to alleviate potential congestion at CSs, by arranging EVs to travel towards lightly congested CSs for charging (meaning they will experience a shorter waiting time). Due to the same reason, the proposed scheme also charges more H-EVs, than the PCWR in Fig. 3(b). Concerning those L-EVs with a lower charging priority, their average waiting time is increased in Fig. 3(c). This is because more L-EVs will be delayed for charging, either if there are still H-EVs locally parked at CSs, or the arrival time of incoming $\mathrm{H}-\mathrm{EV}$ s is earlier than the time to start charging L-EVs. Particularly, the number of charged L-EVs is decreased in Fig. 3(d), since more H-EVs will be charged with a higher priority, where the proposed scheme outperforms PCWR.

\section{CONCLUSION}

In this paper, we have proposed a new spatio-temporal aware and preemptive charging approach for optimal decision making on selecting CSs. It has considered the extra knowledge on both those EVs already parked and waiting for the recharge, and also those still with reasonable distances to CSs and making charging reservations. The extensive numerical studies, based on Helsinki city real geographical and traffic scenarios, have confirmed that our new approach can minimize the charging waiting time for heterogeneous EVs and also accommodate higher number of EVs for charging in one CSs without degrading user's experiences, associated with a consequence of carbon footprint reductions. A more extensive numerical study on the carbon emission reduction because charging time saving is on the way, and a comparison between this protocol and massive simulation studies and more in-depth multi-objective optimization modelling based analytical study are conducted as future work. 


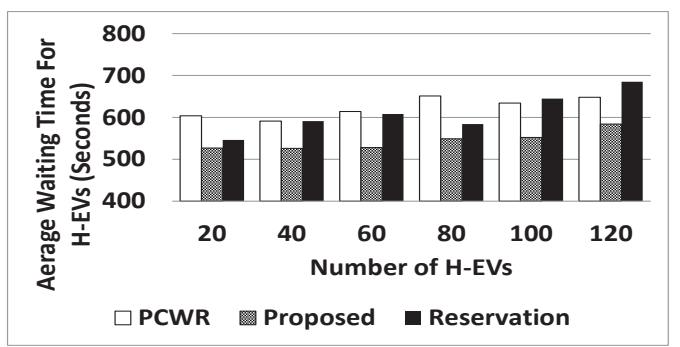

(a) Average Waiting Time for H-EVs

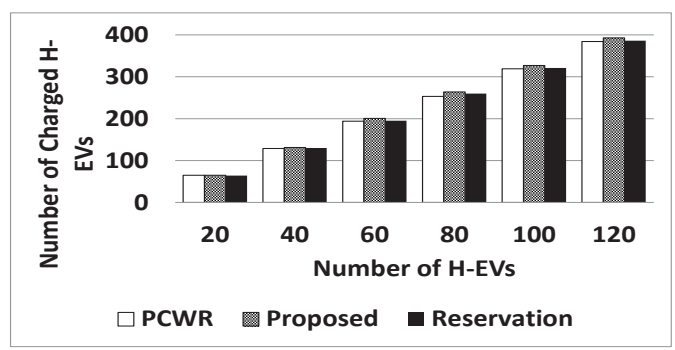

(b) Number of Charged H-EVs

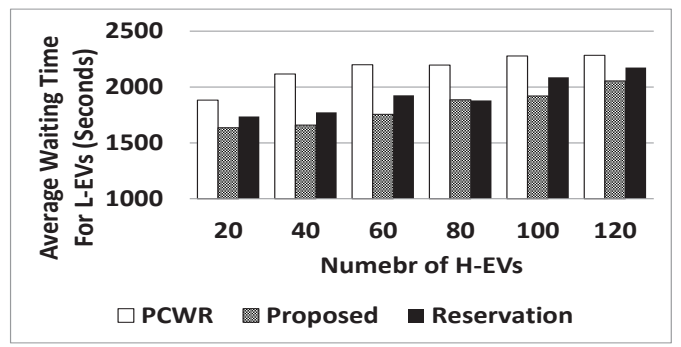

(c) Average Waiting Time for L-EVs

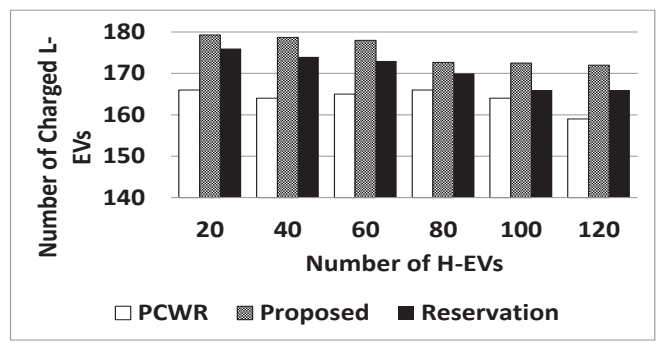

(d) Number of Charged L-EVs

Fig. 3. Influence of H-EVs

\section{REFERENCES}

[1] Y. Cao, O. Kaiwartya, R. Wang, T. Jiang, Y. Cao, N. Aslam, and G. Sexton, "Towards Efficient, Scalable and Coordinated On-the-move EV Charging Management," IEEE Wireless Communications, vol. 24, no. 2, pp. 66-73, 2017.

[2] T. Zhang, W. Chen, Z. Han, and Z. Cao, "Charging Scheduling of Electric Vehicles With Local Renewable Energy Under Uncertain Electric Vehicle Arrival and Grid Power Price," IEEE Transactions on Vehicular Technology, vol. 63, no. 6, pp. 2600-2612, July, 2014.

[3] W. Tang, S. Bi, and Y. Zhang, "Online Coordinated Charging Decision Algorithm for Electric Vehicles Without Future Information," IEEE Transactions on Smart Grid, vol. 5, no. 6, pp. 2810-2824, November, 2014.

[4] P. Rezaei, J. Frolik, and P. Hines, "Packetized Plug-In Electric Vehicle Charge Management," IEEE Transactions on Smart Grid, vol. 5, no. 2, pp. 642-650, March, 2014.

[5] J. Timpner and L. Wolf, "Design and Evaluation of Charging Station Scheduling Strategies for Electric Vehicles," IEEE Transactions on Intelligent Transportation Systems, vol. 15, no. 2, pp. 579-588, April, 2014.
[6] Y. He, B. Venkatesh, and L. Guan, "Optimal Scheduling for Charging and Discharging of Electric Vehicles," IEEE Transactions on Smart Grid, vol. 3, no. 3, pp. 1095-1105, September, 2012.

[7] S.-N. Yang, W.-S. Cheng, Y.-C. Hsu, C.-H. Gan, and Y.-B. Lin, "Charge Scheduling of Electric Vehicles in Highways," Elsevier Mathematical and Computer Modelling, vol. 57, no. 1112, pp. 2873 - 2882, June, 2013.

[8] M. Gharbaoui, L. Valcarenghi, R. Bruno, B. Martini, M. Conti, and P. Castoldi, "An Advanced Smart Management System for Electric Vehicle Recharge," in IEEE IEVC' 2012, Greenville, SC, USA, March, 2012.

[9] Q. Guo, S. Xin, H. Sun, Z. Li, and B. Zhang, "Rapid-Charging Navigation of Electric Vehicles Based on Real-Time Power Systems and Traffic Data," IEEE Transactions on Smart Grid, vol. 5, no. 4, pp. 1969-1979, July, 2014.

[10] Y. Cao, N. Wang, and G. Kamel, "A Publish/Subscribe Communication Framework For Managing Electric Vehicle Charging," Vienna, Austria, November, 2014.

[11] M. de Weerdt, S. Stein, E. Gerding, V. Robu, and N. Jennings, "Intention-Aware Routing of Electric Vehicles," IEEE Transactions on Intelligent Transportation Systems, vol. 17, no. 5, pp. 1472 - 1482, 2015.

[12] K. Yuuko, K. Yamazaki, and M. Wachi, "Expansion of FAST Into Tokyo Metropolitan Area," in ITS World Congress' 13, Tokyo, Japan, 2013.

[13] http://www.standard.co.uk/news/london/scotland-yard-to-adopt-newfleet-of-hybrid-police-cars-and-bikes-to-combat-toxic-air-in-londona3455436.html .

[14] Y. Cao, N. Wang, G. Kamel, and Y.-J. Kim, "An Electric Vehicle Charging Management Scheme Based on Publish/Subscribe Communication Framework," IEEE Systems Journal, vol. PP, no. 99, pp. 1-14, 2015.

[15] H. Qin and W. Zhang, "Charging Scheduling with Minimal Waiting in a Network of Electric Vehicles and Charging Stations," in ACM VANET ' 11, Las Vegas, Nevada, USA, September, 2011.

[16] Y. Cao, X. Zhang, R. Wang, L. Peng, N. Aslam, and X. Chen, "Applying DTN Routing for Reservation-Driven EV Charging Management in Smart Cities," in IEEE IWCMC' 17, Valencia, Spain, June, 2017. 